\title{
Meta-analysis of 20 genome-wide linkage studies evidenced new regions linked to asthma and atopy
}

\author{
Emmanuelle Bouzigon ${ }^{\star, 1,2,3}$, Paola Forabosco ${ }^{4,5}$, Gerard H Koppelman ${ }^{6}$, William OCM Cookson ${ }^{7}$, \\ Marie-Hélène Dizier ${ }^{8,9}$, David L Duffy ${ }^{10}$, David M Evans ${ }^{11}$, Manuel AR Ferreira ${ }^{10}$, Juha Kere ${ }^{12}$, Tarja Laitinen ${ }^{13}$, \\ Giovanni Malerba ${ }^{14}$, Deborah A Meyers ${ }^{15}$, Miriam Moffatt ${ }^{7}$, Nicholas G Martin ${ }^{10}$, Mandy Y Ng ${ }^{16}$, \\ Pier Franco Pignatti ${ }^{14}$, Mathias Wjst ${ }^{17,18}$, Francine Kauffmann ${ }^{9,19}$, Florence Demenais ${ }^{1,2,3}$ and Cathryn M Lewis ${ }^{4,16}$
}

Asthma is caused by a heterogeneous combination of environmental and genetic factors. In the context of GA2LEN (Global Allergy and Asthma European Network), we carried out meta-analyses of almost all genome-wide linkage screens conducted to date in 20 independent populations from different ethnic origins ( $\geq 3024$ families with $\geq 10027$ subjects) for asthma, atopic asthma, bronchial hyper-responsiveness and five atopy-related traits (total immunoglobulin E level, positive skin test response (SPT) to at least one allergen or to House Dust Mite, quantitative score of SPT (SPTQ) and eosinophils (EOS)). We used the genome scan meta-analysis method to assess evidence for linkage within bins of traditionally 30-cM width, and explored the manner in which these results were affected by bin definition. Meta-analyses were conducted in all studies and repeated in families of European ancestry. Genome-wide evidence for linkage was detected for asthma in two regions (2p21-p14 and 6p21) in European families ascertained through two asthmatic sibs. With regard to atopy phenotypes, four regions reached genome-wide significance: 3p25.3-q24 in all families for SPT and three other regions in European families (2q32-q34 for EOS, 5q23-q33 for SPTQ and 17q12-q24 for SPT). Tests of heterogeneity showed consistent evidence of linkage of SPTQ to $3 p 11-3 q 21$, whereas between-study heterogeneity was detected for asthma in 2p22-p13 and 6p21, and for atopic asthma in 1q23-q25. This large-scale meta-analysis provides an important resource of information that can be used to prioritize further fine-mapping studies and also be integrated with genome-wide association studies to increase power and better interpret the outcomes of these studies.

European Journal of Human Genetics (2010) 18, 700-706; doi:10.1038/ejhg.2009.224; published online 13 January 2010

Keywords: asthma; atopy; meta-analysis; linkage scan

\section{INTRODUCTION}

Asthma is a complex and heterogeneous disease resulting from many genetic and environmental factors, with a wide spectrum of clinical manifestations. ${ }^{1}$ Asthma is associated with intermediate biological and physiological phenotypes, including traits related to atopy and inflammation (total serum immunoglobulin E (IgE) levels, specific IgE and skin test reactivity to common aero-allergens and eosinophilia) and those related to lung function (forced expiratory volume in one second, and airway responsiveness).

Considerable efforts have been made over the past 10 years to map the chromosomal location of genes potentially involved in the development of asthma. To date, 20 independent research groups have conducted genome-wide linkage (GWL) scans, mainly for asthma and atopy-related phenotypes, in 24 different populations around the world. ${ }^{2}$ These scans have led to the identification of eight genes by positional cloning and also to a large number of genomic regions that may harbour susceptibility genes. ${ }^{3}$ However, many of these regions did not show strong evidence for linkage in individual studies and/or have not been replicated across studies. Lack of replication may derive from discrepancies in phenotype definition across studies, differences in family ascertainment schemes, differences in analytical methods, insufficient power to detect linkage and genetic heterogeneity of the disease.

Meta-analysis, which involves combining the results of GWL screens, is one approach that can improve the information available from a series of underpowered studies. Thus, meta-analysis can be a powerful tool not only for strengthening the evidence of previously detected linkage regions but also for identifying novel regions in which the genetic effect is too small to be detected in a single study. Meta-analysis cannot completely overcome the problem of genetic

\footnotetext{
IINSERM, U946, Paris, France; ${ }^{2}$ Université Paris Diderot, Paris 7, Institut Universitaire d'Hématologie, Paris, France; ${ }^{3}$ Foundation Jean Dausset-Centre d'Etude du Polymorphisme Humain (CEPH), Paris, France; ${ }^{4}$ King's College London, Department of Medical and Molecular Genetics, London, UK; ${ }^{5}$ Istituto di Genetica delle PopolazioniCNR, Alghero, Italy; ${ }^{6}$ Department of Pediatric Pulmonology and Pediatric Allergology, University Medical Center Groningen, University of Groningen, Groningen, The Netherlands; ${ }^{7}$ National Heart Lung Institute, Imperial College, London, UK; ${ }^{8}$ INSERM, U535, Villejuif, France; ${ }^{9}$ Univ Paris-Sud, IFR69, Villejuif, France; ${ }^{10}$ Queensland Institute of Medical Research, Royal Brisbane Hospital, Brisbane, Australia; ${ }^{11}$ Department of Social Medicine, University of Bristol, Bristol, UK; ${ }^{12}$ Department of Biosciences and Nutrition, Karolinska Institute, Stockholm, Sweden; ${ }^{13}$ Departments of Pulmonary Medicine and Medical Genetics, University of Helsinki, Helsinki, Finland; ${ }^{14}$ Section of Biology and Genetics, Department of Mother and Child, and Biology-Genetics, University of Verona, Verona, Italy; ${ }^{15}$ Center for Human Genomics, Wake Forest University, Winston Salem, NC, USA; 16King's College London, MRC SGDP Centre, Institute of Psychiatry, London, UK; ${ }^{17}$ Institute of Experimental Pneumology, Helmholtz Zentrum München, German Research Center for Environmental Health, Neuherberg, Germany; ${ }^{18}$ Institute of Genetic Medicine, EURAC research, Bozen, Italy; ${ }^{19}$ INSERM, U780, Villejuif, France

*Correspondence: Dr E Bouzigon, INSERM U946, Fondation Jean Dausset-CEPH, 27 rue Juliette Dodu, 75010 Paris, France. Tel: +33 1 53725023; Fax: +33 1 53725049; E-mail: emmanuelle.bouzigon@inserm.fr

Received 12 May 2009; revised 29 September 2009; accepted 12 November 2009; published online 13 January 2010
} 
heterogeneity but can identify regions implicated in a subset of the pooled studies. Regions highlighted in meta-analysis of linkage can be useful to prioritize future gene localization studies, whether these are based on positional cloning or on follow-up of genome-wide association (GWA) studies. Moreover, it has been shown that taking into account previous linkage information while computing the falsediscovery rate can improve the power of GWA studies. ${ }^{4}$ Recently, a meta-analysis of nine genome scans conducted in families of European ancestry (EUR) for asthma and three asthma-associated phenotypes (IgE, positive skin test response and bronchial hyper-responsiveness (BHR)) identified several regions with suggestive evidence of linkage, but failed to reach a genome-wide significance level. ${ }^{5}$

In the context of GA2LEN (Global Allergy and Asthma European Network, http://www.ga2len.net), we conducted a collaborative meta-analysis of $20 \mathrm{GWL}$ scans conducted worldwide for the most studied phenotypes across studies, namely, asthma, atopic asthma, BHR and five atopy-related phenotypes, using the genome scan meta-analysis (GSMA) method. ${ }^{6}$ The inclusion of populations with different genetic ancestry allowed us to identify common genetic regions underlying the pathogenesis of asthma across populations and to evaluate the variability of linkage findings by formally testing for genetic heterogeneity among studies. ${ }^{7}$ We also explored the robustness of our results by using different bin-width definitions for the GSMA method.

\section{MATERIALS AND METHODS}

\section{Study inclusion criteria}

We identified 24 GWL studies conducted for asthma or asthma-associated phenotypes, using PubMed searches (last search updated January 2009, using the following keywords: 'asthma', 'atopy', 'BHR', 'IgE', 'eosinophils', 'skin tests', 'genome search', 'genome scan') and by examining reference lists of papers in asthma genetics. Results were limited to English language papers. This literature search was conducted by the first author (EB), and the inclusion of data and decision strategy were reviewed by the statistical genetics group (EB, PF, FD and $\mathrm{CML}$ ). We excluded linkage studies of candidate regions, which only considered short regions of the genome. Linkage studies that overlapped across samples or were extended versions of previous publications were identified, and only independent studies were included. In cases in which studies had performed a two-stage analysis that genotyped more markers in targeted regions at stage 2, only stage 1 results were used, as GSMA requires a uniform distribution of markers and families across the genome.

Among the 24 eligible GWL scans, 20 were included in this study. The four excluded studies included two studies focusing on specific regions (positional cloning studies) that mentioned in their publication that they had conducted GWL screens but without publishing their genome-wide results, ${ }^{8,9}$ one study focusing on specific IgE that was not a phenotype we examined ${ }^{10}$ and one study with no data that could be made available for our meta-analysis. ${ }^{11}$ As described in Table 1, for 12 of 20 included studies, we obtained data on marker maps and, for each phenotype, GWL statistics (eg, LOD or NPL scores and/or $P$-values) from investigators ${ }^{12-20}$ or from a website (http://www.springerlink.com/ content/8660v8526j362846/MediaObjects/439_2006_285_MOESM2_ESM.xls). ${ }^{21}$

Table 1 Characteristics of individual genome-wide linkage scans included in this study

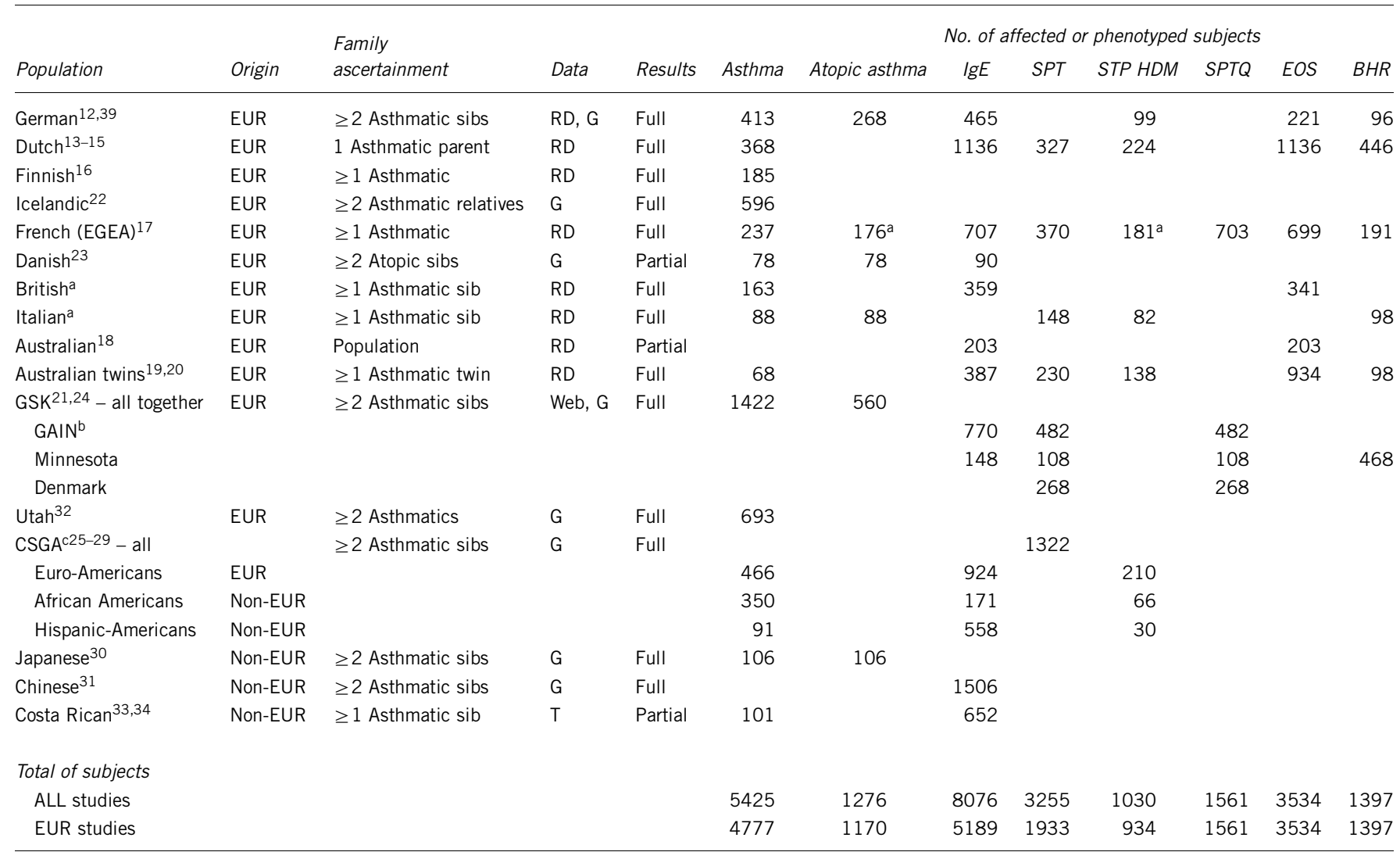

Abbreviations: SPT, defined by at least one positive skin prick test response; SPT HDM, positive skin prick test response to House Dust Mite; SPTQ, quantitative measure of positive skin test response to allergens; EOS, eosinophil count; BHR, bronchial hyper-responsiveness; RD, raw data available; G, data extracted from published graphs; Web, data available online; T, published table. response to allergens; EOS, eosinophil count; BHR, bronchial hyper-responsiveness; RD, raw data available; G, data extracted from published graphs; Web, data available
aUnpublished results.

bIgE results for GAIN and Minnesota groups were included separately; atopy results were included separately for GAIN, Minnesota and Denmark families, respectively.

${ }^{C}$ Asthma and IgE were analyzed separately in families of European, African-American and Hispanic-American origin. 
For seven studies, results were presented as chromosomal graphs from multipoint linkage analysis, and linkage scores were extracted using the digitizing program Engauge Digitiser (v.2.14, Mark Mitchell, 2002, http://digitizer. sourceforge.net/), which converts curves into $x$ and $y$ coordinates. ${ }^{22-32}$ Finally, for one study, we used data extracted from published tables, in which only peak linkage statistics were shown.

\section{Phenotypes analyzed}

The following eight phenotypes were considered by this meta-analysis: asthma, atopic asthma, BHR, a positive skin prick test response to at least one allergen (SPT) or specifically to House Dust Mite (SPT to HDM), a quantitative measure of positive skin test response to allergens (SPTQ, corresponding to the proportion of positive skin prick tests), total serum IgE levels and eosinophil (EOS) counts. Asthma definition was based on a physician's diagnosis in six studies, on standardized questionnaires in four studies or on self-reporting and/or doctor diagnosed combined with different traits (BHR and/or asthma therapy and/or hospitalization for asthma) in five studies. Finally, asthma was based on a decision algorithm in the Dutch study. ${ }^{15}$ The atopic asthma phenotype was defined by adding the presence of positive SPT (seven studies) and/or specific IgE (one study) to the previous definitions of asthma. The BHR definition was based on methacholine (four studies) or histamine challenge tests (two studies).

\section{Genome search meta-analysis}

Results obtained from individual GWL scans were combined using the GSMA method. ${ }^{6,35}$ The GSMA method is a rank-based analysis assessing the strongest evidence for linkage within bins of fixed width, traditionally $30 \mathrm{cM}$, resulting in a total of 118 bins on the autosomes using the Marshfield map. For each study, the maximum evidence of linkage achieved within a bin was noted (eg, maximum LOD or NPL or minimum $P$-values). When partial data were available, bins without results were all assigned a rank equal to the median of unused ranks. Bins were then ranked and ranks were summed across studies. Statistical evidence for linkage within the bin was assessed from this summed rank (SR) using permutation tests available in the GSMA software. $^{36}$ By applying Bonferroni's correction for multiple testing on the basis of 118 bins, a $P$-value of $0.05 / 118=0.00042$ is necessary for genome-wide evidence of linkage, and a $P$-value of $1 / 118=0.0084$ for suggestive evidence of linkage.

For each phenotype, a meta-analysis was performed for all studies, and repeated for the sub-sample consisting of only families with EUR ancestry. Initially, only linkage screens with genome-wide results were analyzed, thereafter genome scans with partial results were added. Meta-analyses of the different traits were performed both unweighted (assuming equal contribution from each study) and weighted by study size, using the square root of the number of affected individuals (binary traits) or phenotyped subjects (quantitative traits) as a weighting factor. To explore the manner in which meta-analysis results could be affected by bin definition, we also performed analyses using $20 \mathrm{cM}$ (giving a total of 173 bins) and $40 \mathrm{cM}$ (87 bins) bin widths, and shifted $30-\mathrm{cM}$ bins obtained by moving bin boundaries by $15 \mathrm{cM} .{ }^{37}$ Multiple testing of bins was accounted for in the $P$-value thresholds used, but multiple testing of phenotypes was not corrected for as these phenotypes are correlated. The outcomes of sensitivity analysis according to bin width were only examined in regions detected by the original $30-\mathrm{cM}$ bin-width analysis.

\section{Testing for between-scan heterogeneity}

In bins reaching the $5 \%$ threshold value, we tested for heterogeneity (1) among ALL studies and (2) among EUR studies, using the $Q$-statistic proposed by Zintzaras and Ioannidis. ${ }^{7}$ For bin $j, Q=\sum_{i=1}^{N} w_{i}\left(R_{i, j}-R_{j}\right)^{2}$, where $w_{i}$ is the weight of study $i, R_{i, j}$ the rank of study $i$ in bin $j$ and $R_{j}$ the mean rank for bin $j$. The statistical significance of the heterogeneity metric was tested using Monte Carlo permutations, that is, by randomly reassigning the ranks to bins within each study, and recalculating each heterogeneity statistic. High between-study heterogeneity was defined as the proportion of simulated bins with Q-statistics exceeding the observed value, indicating large differences in the relative rank of a bin among studies. Conversely, low heterogeneity was defined as the percentage of simulated bins below the observed value, corresponding to consistent linkage evidence across studies. High or low between-study heterogeneity was based on one-sided tests $(P<0.05$ for left- or right-sided testing, respectively). As the distribution of the heterogeneity statistic depends on the SR statistic, ${ }^{7}$ the observed heterogeneity statistic was only compared with simulated bins with similar SR values $( \pm 2)$. Heterogeneity tests were performed using HEGESMA software. ${ }^{38}$

\section{RESULTS}

In total, our meta-analysis included genome linkage scan results on asthma and atopy-related phenotypes from 20 different studies (including more than 3000 families). Genome screens were carried out in families with different geographical and ethnic origins: Europe, United States (families of European, African-American and Hispanic-American origin), Australia, Japan, China and Costa Rica (Table 1). Families were mainly ascertained through at least one asthmatic subject, with some additional affected individuals available. All linkage analyses, except two, were multipoint nonparametric, using a range of programs (eg, Mapmaker/Sibs, GeneHunter, Solar, MLB-GH, Merlin) reflecting family structures and date of the analysis.

The main results from weighted ( $\left.\mathrm{p}_{\mathrm{SR}} \mathrm{W}\right)$ and unweighted ( $\left.\mathrm{p}_{\mathrm{SR} \_\mathrm{U}}\right)$ meta-analyses (30-cM bin width) conducted in the entire set of studies (ALL families) and in EUR families are shown in Table 2 and in Supplementary Table S1. Figure 1 and Supplementary Figure S1 illustrate the results for all bins (ALL and EUR analyses). Exhaustive meta-analysis results (eight phenotypes) are presented in Supplementary Tables S2-S10.

\section{Asthma and atopic asthma}

Meta-analysis of asthma was performed in 16 asthma GWL studies, of which 14 scans had complete results (2053 families with 5425 affected individuals). Suggestive evidence for linkage occurred in two bins: bin $4 \_7\left(p_{S R \_W}=0.007\right)$ and bin 5_5 $\left(\mathrm{p}_{\mathrm{SR} \_\mathrm{W}}=0.003\right)$ in ALL families. Moreover, irrespective of the strategy used (different bin width, inclusion of two scans with partial results), suggestive evidence of linkage was consistently detected on chromosome 4 between 182 and $200 \mathrm{cM}$ and on chromosome 5 between 139 and $141 \mathrm{cM}$. When restricting the analysis to studies including families ascertained through at least two asthmatic sibs, suggestive evidence of linkage was observed in bin 2_3 ( $\left.\mathrm{p}_{\mathrm{SR} \_\mathrm{W}}=0.002\right)$ and bin 6_2 $\left(\mathrm{p}_{\mathrm{SR} \_\mathrm{W}}=0.004\right)$, both of which reached genome-wide significance level ( $\mathrm{p}_{\text {SR_W }}$ $<0.0002$ ) in EUR families. Varying bin widths (Supplementary Figure S2) supported these results and defined two linked regions of <20 cM: 2p21-p14 (63.4-82.8cM) and 6p21.31-p21.1 (49.5$65.1 \mathrm{cM})$. Genetic heterogeneity $\left(\mathrm{p}_{\mathrm{SR} \_\mathrm{W}} \geq 0.975\right)$ between studies was detected in ALL families for bin 2_3 and in families ascertained through two asthmatic sibs for bin $6 \_2$.

Overall, data for atopic asthma consisted of 618 families with 1276 atopic asthmatic offspring from six studies. Suggestive evidence of linkage was detected on chromosome $17 \mathrm{q}$ in ALL families and when analyses were restricted to EUR families (bin 17_3, $\left.\mathrm{p}_{\mathrm{SR} \_} \mathrm{W}_{\mathrm{H}}=\mathrm{p}_{\mathrm{SR}_{-} \mathrm{U}}=0.001\right)$. Analyses with different bin-width definitions consistently detected the $17 \mathrm{q}$ region at a suggestive evidence level ( $\mathrm{p}_{\mathrm{SR} \_\mathrm{W}}$ varying from 0.0004 to 0.001 for ALL families and from 0.0006 to 0.002 in the EUR sample), delineating a linked region of $18 \mathrm{cM}$ between 63 and $81 \mathrm{cM}$. Suggestive evidence for linkage was also obtained for two other regions: $1 \mathrm{p}\left(125-145 \mathrm{cM}, \mathrm{p}_{\mathrm{SR}} \mathrm{W}=0.006\right)$ and $1 \mathrm{q}\left(271 \mathrm{cM}\right.$-qter, $\left.\mathrm{p}_{\mathrm{SR}} \mathrm{W}=0.005\right)$ in EUR families. For the $1 \mathrm{q}$ region, there was a nominally significant evidence for genetic heterogeneity between studies $\left(\mathrm{p}_{\mathrm{SR} \_\mathrm{W}} \geq 0.98\right)$, for both $\mathrm{ALL}$ and EUR families. 


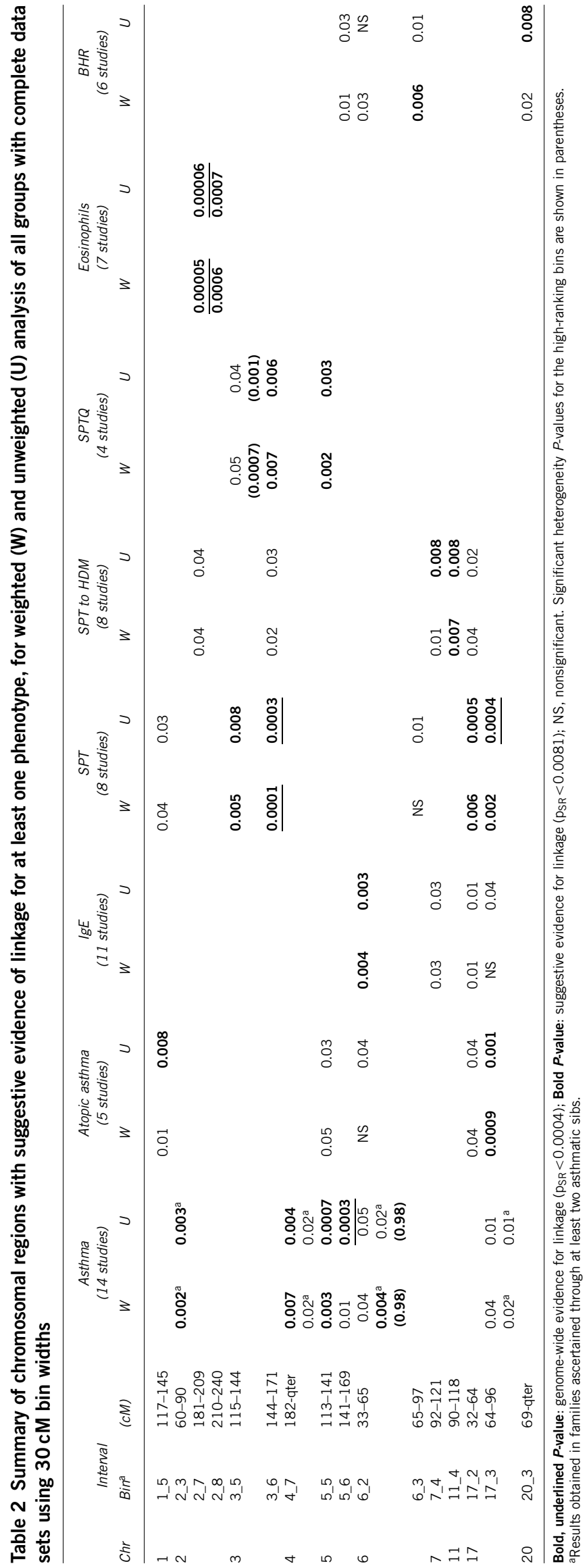

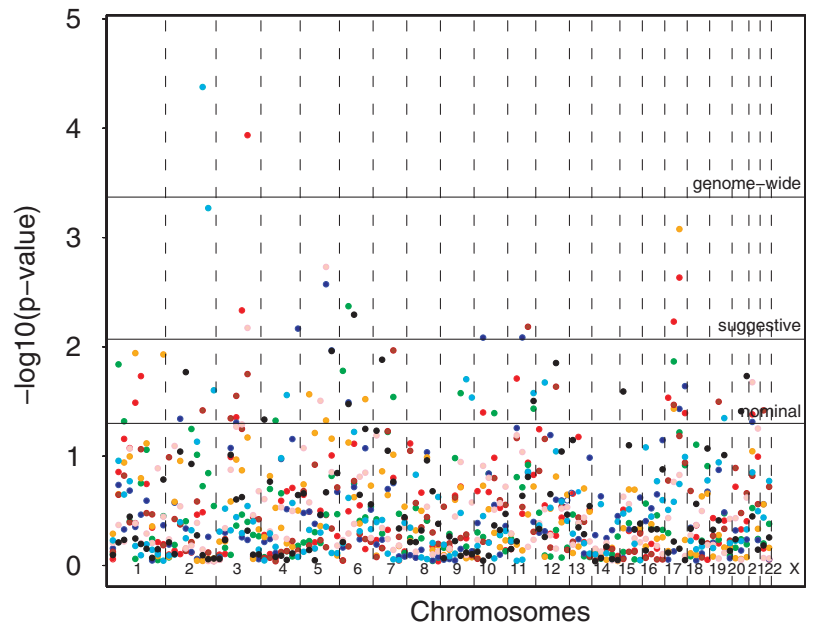

Figure 1 Meta-analysis results for genome-wide linkage scans of asthma and asthma-related phenotypes in ALL families. Minus Log10 $P$-values of weighted summed ranks (vertical axis) are plotted against the bin location (horizontal axis), with a single point plotted for each bin. Thresholds for genome-wide, suggestive and nominal significance are shown. Each phenotype is represented with a different colour: asthma (blue), atopic asthma (orange), BHR (black), IgE (green), SPT (red), SPT to HDM (brown), SPTQ (magenta) and eosinophils (cyan).

\section{Bronchial hyper-responsiveness}

Meta-analysis for BHR was carried out on six scans, including 391 EUR families (1397 affected subjects). Suggestive evidence for linkage was observed on chromosome 6 (bin 6_3, $\mathrm{p}_{\mathrm{SR} \_\mathrm{W}}=0.006$ ). Varying bins allowed defining a linked region of $13 \mathrm{cM}$ between 65 and $78 \mathrm{cM}$.

\section{IgE levels}

The meta-analysis of 13 GWL scans with available data for IgE included 2471 families, corresponding to 8076 subjects. Suggestive evidence for linkage was obtained in ALL families on chromosome $6 \mathrm{p}$ for bin $6 \_2\left(p_{S R ~}=0.005 ; p_{S R ~}=0.003\right)$. Suggestive evidence for linkage was consistently detected for the $6 \mathrm{q}$ region using different bin widths and/or when adding the Australian population-based scan with partial results $\left(\mathrm{p}_{\mathrm{SR}}\right.$ between 0.001 and 0.01 ). When restricting the study to EUR families, no region reached the suggestive significance threshold.

\section{SPT phenotypes}

Together, the data for SPT consisted of 1264 families (3255 atopic subjects) from nine scans. Genome-wide evidence for linkage was observed for chromosome 3q (bin 3_6, $\mathrm{p}_{\mathrm{SR} \_\mathrm{W}}=0.0001$ and $\left.\mathrm{p}_{\mathrm{SR}} \mathrm{U}=0.0003\right)$ in ALL families and for two adjacent bins on chromosome $17 \mathrm{q}$ in EUR families: bin 17_2 $\left(\mathrm{p}_{\mathrm{SR} \_\mathrm{W}}=\mathrm{p}_{\mathrm{SR} \_\mathrm{U}}=0.00006\right)$ and bin $17 \_3\left(p_{S R \_W}=0.00009\right.$ and $\left.p_{S R \_U}=0.0001\right)$. Analyses using different bin widths supported the evidence for linkage across a broad region of chromosome $17 \mathrm{q}$ in EUR families and to chromosome $3 \mathrm{q}$ ( $\mathrm{p}_{\mathrm{SR}} \mathrm{W}$ varying from 0.0001 to 0.004 ) in ALL families, defining two large regions of at least $40 \mathrm{cM}$ between 124 and $166 \mathrm{cM}$ for chromosome 3 and between 43 and $86 \mathrm{cM}$ for chromosome 17 (Supplementary Figure S2).

The meta-analysis for SPT to HDM included eight scans with a total of 548 families (1030 affected subjects). Weighted analysis identified a single region with suggestive evidence of linkage: bin $11 \_4\left(90-118 \mathrm{cM}, \mathrm{p}_{\mathrm{SR}}=0.007\right)$. 
Meta-analysis for a quantitative score of SPT (SPTQ) was performed on four scans consisting of only EUR families (715 families with 1561 offspring). Suggestive evidence of linkage was observed for two bins: bin 5_5 ( $\left.p_{S R} \leq 0.003\right)$ and bin $3 \_6\left(p_{S R} \leq 0.007\right)$. Analyses using different bin widths (Supplementary Figure S2) delineated a minimal region of $\sim 28 \mathrm{cM}$ on chromosome 5q23.1-q33.1 with genome-wide evidence of linkage $\left(128-156 \mathrm{cM}, \mathrm{p}_{\mathrm{SR}}=0.0002\right)$. Genetic heterogeneity between studies was detected for bin 21_1 $\left(\mathrm{p}_{\mathrm{SR} \_\mathrm{W}}=0.975\right)$. Conversely, bin 3_5 showed consistent evidence of linkage across studies $\left(\mathrm{p}_{\text {SR_W }} \leq 0.001\right)$.

\section{Eosinophils}

The data for EOS included six GWL scans with 1285 EUR families, corresponding to 3534 subjects. Genome-wide evidence of linkage occurred on the $2 \mathrm{q} 31.1-\mathrm{q} 34$ region irrespective of the strategy used for bin 2_7 $\left(181-210 \mathrm{cM}, \mathrm{p}_{\mathrm{SR}}=5.10^{-5}\right)$, with suggestive evidence of linkage in the adjacent bin $2 \_8\left(210-241 \mathrm{cM}, \mathrm{p}_{\mathrm{SR}}=0.0006\right)$. Varying bin widths supported these results and defined a linked region of $15 \mathrm{cM}$ between 194 and $210 \mathrm{cM}$ (2q32.3-q34) (Supplementary Figure S2).

\section{DISCUSSION}

This meta-analysis of 20 genome linkage scans conducted for asthma, BHR and five atopy-related phenotypes has identified several chromosomal regions that are likely to harbour genes involved in asthma. This study has provided genome-wide evidence of linkage to five regions in European families (2p21-p14 and 6p21 for asthma, 2q31.1q34 for EOS, 5q23.1-q33.1 for SPTQ, 17p12-q25 for SPT) and to one region in all families (3p25.3-q24 with SPT). Tests of heterogeneity indicated consistent evidence of linkage of SPTQ to the 3p11-3q21 region and between-study heterogeneity for asthma for 2p21-p14 and $6 \mathrm{p} 21$, and for atopic asthma for 1q23-q25.

We can first compare our results with those of the recent GSMA study performed by Denham et al..$^{5}$ Whereas the main phenotypes (asthma, SPT, IgE and BHR) have been examined by both metaanalyses, this study explored the atopic dimension of asthma in more detail (atopic asthma, polysensitization, positive skin test to HDM and EOS) and included all genome-wide scans conducted to date. There are a number of consistencies in the results of the two meta-analyses. Regions identified at a suggestive significance level by Denham et al were detected in this study with at least the same significance level, and three of them reached genome-wide significance (chromosomes 6 for asthma, 3 and 17 for SPT) in our meta-analysis. Moreover, we identified additional regions with genome-wide or suggestive evidence of linkage: 2p21-p14, 4q34.3-qter and 5q31.1-5q31.2 regions for asthma. The differences in identified regions and significance levels were likely to be partly due to the differences in population included in the two meta-analyses. Indeed, Denham et al selected nine genome-wide scans conducted in EUR families, whereas we collected 20 genome scans performed in various ethnic populations, including European, Hispanic, Asian and African-American subjects. This strategy allowed searching for common genetic regions across populations and testing for genetic heterogeneity between studies. We also included two unpublished European studies, but sensitivity analyses showed that these two data sets did not influence our findings. There are also methodological differences between published and the present meta-analyses: we used only scans with full data in our primary analysis, and then added those with partial data, and we used different bin definitions to conduct sensitivity analyses. We also conducted heterogeneity tests and evidenced genetic heterogeneity between studies in four linkage regions.
In this study, we performed GSMA on genome-wide scans for asthma-related phenotypes using the traditional $30-\mathrm{cM}$ bin-width definition that allows having at least two bins of equal width on the smallest chromosomes. However, peak linkage scores in individual studies may map to adjacent bins, and dilute the evidence for linkage in the GSMA study, reducing both power and precision. To evaluate the consistency of our results, we repeated the analyses using two additional bin widths of 20 and $40 \mathrm{cM}$ and using a shifted $30-\mathrm{cM}$ bin. ${ }^{37}$ Significant results were obtained consistently for regions identified at genome-wide or suggestive significance levels ( $2 p$ and $6 p$ with asthma, $2 \mathrm{q}$ with EOS, $17 \mathrm{q}$ with atopic asthma, $3 \mathrm{q}$ and $17 \mathrm{q}$ regions with SPT and $6 \mathrm{p}$ with IgE). These analyses strengthened the evidence of linkage to the $5 \mathrm{q}$ region for SPTQ and identified new regions for atopic asthma (1p22-p13, 1q23-q25) and SPT (1p34-p22). Moreover, these analyses allowed us to delineate the chromosomal regions of interest that are to be further investigated.

Regarding asthma, our meta-analysis allowed the identification of a novel region (2p21-p14). Considering the contribution to the $2 \mathrm{p}$ locus among all studies individually, six scans only showed weak evidence of linkage to this locus $(P \geq 0.01)$. This suggests that the genetic effect mediating the linkage may be too weak to be identified by any of the individual scans and highlights the importance of conducting a metaanalysis to identify such a candidate region. Moreover, between-study heterogeneity was significantly high. Such heterogeneity may be due to differences in family ascertainment and may also reflect the intrinsic complexity of the disease architecture or differences in asthma definition across studies. Indeed, asthma genetic research is hampered by the lack of a gold standard for case definitions, and linkage studies are sensitive to the misclassification of individuals, leading to reduction of statistical power. However, we failed to identify a relationship between the detected heterogeneity for chromosome 2 and variation in asthma definition. Interestingly, analysis of different bin widths delineated a chromosomal region $(63-83 \mathrm{cM})$ that overlaps a locus identified for severe asthma in German families ${ }^{39}$ and is located at $10 \mathrm{cM}$ from a linkage signal detected in the French EGEA study for an asthma severity score. ${ }^{40}$

For SPT, the strongest evidence of linkage in the analysis of all families arose in the 3p25.3-q24 region. This evidence was not sustained when restricting the analysis to EUR families, suggesting that this region may harbour a major susceptibility gene that is involved in all populations. Indeed, among the nine genome scans included in the meta-analysis, two had suggestive evidence of linkage, five scans having LOD $>0.70$. This region has been reported to be linked to various atopy (IgE, specific IgE, EOS) and lung function phenotypes, ${ }^{17,19,23,34,41,42}$ and it harbours the COL29A1 gene that was recently found to be associated with atopic dermatitis, ${ }^{43}$ as well as genes encoding pattern recognition receptors (TLR9) and chemokine receptors (CCR3, CCR5).

The most significant result for SPT obtained from GSMA in families of EUR was the 17p12-q25 region. Significant or suggestive linkage to this region was detected in four studies (French, Dutch, Australian twin and GAIN), ${ }^{14,17,20,24}$ but not in others, confirming the ability of GSMA to detect linkage in the presence of heterogeneity. Interestingly, evidence of linkage of the $17 \mathrm{q}$ region was less significant when considering the whole sample, including African-American and Hispanic-American families from the CSGA study, suggesting that susceptibility gene(s) included in that region may have a more important role in Caucasian populations. Recently, the first GWA study conducted for asthma in European children identified a strong association of the disease with the $17 \mathrm{q} 21$ locus that harbours GSDML and ORMDL3 genes. ${ }^{44}$ These genes are located at the boundary between 
$17 \_2$ and 17_3 bins. Further studies have shown that the 17q21 genetic variants are more strongly associated with early-onset asthma but did not show association with atopy. ${ }^{45}$ It remains to be investigated whether polymorphisms at the $17 \mathrm{q} 21$ locus account fully or partially for the observed linkage in this region. Moreover, bins on chromosome 17 were detected to be linked to various atopy phenotypes by the present meta-analysis (IgE, EOS, SPT to HDM, asthma and atopic asthma), and linkage signals in this region have also been reported for asthma by previous studies, ${ }^{12,14,17,20,34,46}$ suggesting that this region is likely to harbour several susceptibility genes, a few of them with pleiotropic effects on correlated phenotypes.

The 5q23.1-q33.1 region provided genome-wide evidence of linkage to SPTQ in different analyses. This linkage was supported by three scans with LOD $>1.2$, the fourth one having a modest signal in that region. The $5 \mathrm{q}$ region was also identified by the asthma analysis, this linkage signal being mainly supported by the three smallest studies (Japanese, Australian twins and Hispanic families from the CSGA study) and by the Dutch genome scan. This region was first reported to be linked to $\operatorname{IgE}$ and BHR by candidate linkage studies, and then detected with asthma, suggesting that this region may harbour several genes. ${ }^{3}$ Candidate genes are present within this region, including the $A D R B 2$ gene and the interleukin gene cluster. ${ }^{3}$ Moreover, this region includes the CYFIP2 gene found to be associated with atopic asthma by positional cloning. ${ }^{47}$

Genome-wide evidence of linkage was detected on $2 \mathrm{q} 32-\mathrm{q} 34$ for EOS. This region is reported to be linked to IgE and lung function ${ }^{12,41}$ and harbours several candidate genes, including genes involved in T-cell signalling (ICOS, CD28 and CTLA-4) and apoptosis (CASP8). This region includes the IL1R1L gene found to be associated with EOS by a GWA study. ${ }^{48}$

Suggestive evidence was also observed on chromosome 6p22-p21 for IgE and asthma. No individual study showed strong evidence of linkage with IgE (LOD > 2.0) in this region, but six had nominally significant LOD scores (LOD $>0.70$ ). This $6 \mathrm{p}$ region was mainly detected to be linked to asthma and atopy-related phenotypes. ${ }^{14,18,23,25,30,49}$ This chromosomal region includes the MHC region and in particular the HLA-G locus found to be associated with asthma and BHR in an isolated population not included in the present analysis and replicated in Dutch families included in the present meta-analysis. ${ }^{50}$

GWA studies have successfully identified novel genes contributing to common complex diseases but which, however, explain a small proportion of heritability of disease. ${ }^{51}$ It is more and more accepted now that genetic susceptibility to complex diseases such as asthma includes a wide spectrum of genetic variation, from rare alleles with strong effects to common alleles with modest effects. ${ }^{52}$ Current GWA studies have the power to identify mainly common variants, whereas linkage analysis can detect different types of genetic factors within one locus or several loci, including rare variants segregating in families. Therefore, our meta-analysis provides an important resource of information that can be used not only to prioritize further fine-mapping studies in regions of interest but also be integrated with GWAs to increase power ${ }^{4}$ and better interpret the outcomes of these studies.

\section{CONFLICT OF INTEREST}

The authors declare no conflict of interest.

\section{ACKNOWLEDGEMENTS}

We thank all patients who participated in the different studies. We also thank Professors William Cookson and Pier Franco Pignatti and the EGEA cooperative group for providing unpublished results. This study was supported by the European Commission as part of the GA2LEN project, Global Allergy and Asthma European Network (contract no. FOOD-CT-2004-506378) and GABRIEL, a multidisciplinary study to identify the genetic and environmental causes of asthma in the European Community (contract no. 01896 under the Integrated Programme LSH-2004-1.2.5-1 Postgenomic approaches to understand the molecular bias of asthma aiming at a preventive or therapeutic control). This research was funded by a grant from the UK MRC to Cathryn M Lewis (G0400960). The Finnish study was supported by the Academy of Finland and Sigrid Jusélius Foundation. The Dutch family study was supported by grants from the Netherlands Asthma Foundation (AF 95.09 and 98.48) and the US National Institute of Health.

1 Wenzel SE: Asthma: defining of the persistent adult phenotypes. Lancet 2006; 368 : 804-813.

2 Steinke JW, Rich SS, Borish L: 5. Genetics of allergic disease. J Allergy Clin Immuno 2008; 121: S384-S387.

3 Vercelli D: Discovering susceptibility genes for asthma and allergy. Nat Rev Immunol 2008; 8: 169-182.

4 Roeder K, Bacanu SA, Wasserman L, Devlin B: Using linkage genome scans to improve power of association in genome scans. Am J Hum Genet 2006; 78: 243-252.

5 Denham S, Koppelman GH, Blakey J et al: Meta-analysis of genome-wide linkage studies of asthma and related traits. Respir Res 2008; 9: 38.

6 Wise LH, Lanchbury JS, Lewis CM: Meta-analysis of genome searches. Ann Hum Genet 1999; 63: 263-272.

7 Zintzaras E, loannidis JP: Heterogeneity testing in meta-analysis of genome searches. Genet Epidemiol 2005; 28: 123-137.

8 Van Eerdewegh P, Little RD, Dupuis J et al: Association of the ADAM33 gene with asthma and bronchial hyperresponsiveness. Nature 2002; 418: 426-430.

9 Balaci L, Spada MC, Olla N et al: IRAK-M is involved in the pathogenesis of early-onset persistent asthma. Am J Hum Genet 2007; 80: 1103-1114.

10 Kurz T, Altmueller J, Strauch $\mathrm{K}$ et al: A genome-wide screen on the genetics of atopy in a multiethnic European population reveals a major atopy locus on chromosome 3q21.3. Allergy 2005; 60: 192-199.

11 Ober C, Cox NJ, Abney M et al: Genome-wide search for asthma susceptibility loci in a founder population. The Collaborative Study on the Genetics of Asthma. Hum Mol Genet 1998; 7: 1393-1398.

12 Wjst M, Fischer G, Immervoll T et al: A genome-wide search for linkage to asthma. German Asthma Genetics Group. Genomics 1999; 58: 1-8.

$13 \mathrm{Xu}$ J, Postma DS, Howard TD et al: Major genes regulating total serum immunoglobulin E levels in families with asthma. Am J Hum Genet 2000; 67: 1163-1173.

14 Koppelman GH, Stine OC, Xu J et al: Genome-wide search for atopy susceptibility genes in Dutch families with asthma. J Allergy Clin Immunol 2002; 109: 498-506.

15 Meyers DA, Postma DS, Stine OC et al: Genome screen for asthma and bronchial hyperresponsiveness: interactions with passive smoke exposure. I Allergy Clin Immunol 2005; 115: 1169-1175.

16 Laitinen T, Daly MJ, Rioux JD et al: A susceptibility locus for asthma-related traits on chromosome 7 revealed by genome-wide scan in a founder population. Nat Genet 2001; 28: 87-91.

17 Bouzigon E, Dizier MH, Krahenbuhl C et al: Clustering patterns of LOD scores for asthma-related phenotypes revealed by a genome-wide screen in 295 French EGEA families. Hum Mol Genet 2004; 13: 3103-3113.

18 Daniels SE, Bhattacharrya S, James A et al: A genome-wide search for quantitative trait loci underlying asthma. Nature 1996; 383: 247-250.

19 Evans DM, Zhu G, Duffy DL, Montgomery GW, Frazer IH, Martin NG: Major quantitative trait locus for eosinophil count is located on chromosome 2q. J Allergy Clin Immunol 2004; 114: 826-830.

20 Ferreira MA, O'Gorman L, Le Souef $\mathrm{P}$ et al: Robust estimation of experiment-wise $P$ values applied to a genome scan of multiple asthma traits identifies a new region of significant linkage on chromosome 20q13. Am J Hum Genet 2005; 77: 1075-1085.

21 Pillai SG, Chiano MN, White NJ et al: A genome-wide search for linkage to asthma phenotypes in the genetics of asthma international network families: evidence for a major susceptibility locus on chromosome 2p. Eur J Hum Genet 2006; 14: 307-316.

22 Hakonarson $\mathrm{H}$, Bjornsdottir US, Halapi E et al: A major susceptibility gene for asthma maps to chromosome 14q24. Am J Hum Genet 2002; 71: 483-491.

23 Haagerup A, Bjerke T, Schiotz PO, Binderup HG, Dahl R, Kruse TA: Asthma and atopy - a total genome scan for susceptibility genes. Allergy 2002; 57: 680-686.

24 Webb BT, van den Oord E, Akkari A et al: Quantitative linkage genome scan for atopy in a large collection of Caucasian families. Hum Genet 2007; 121: 83-92.

$25 \mathrm{Xu}$ J, Meyers DA, Ober C et al: Genome-wide screen and identification of gene-gene interactions for asthma-susceptibility loci in three U.S. populations: collaborative study on the genetics of asthma. Am J Hum Genet 2001; 68: 1437-1446.

26 Colilla S, Nicolae D, Pluzhnikov A et al: Evidence for gene-environment interactions in a linkage study of asthma and smoking exposure. J Allergy Clin Immunol 2003; 111: $840-846$ 
27 Mathias RA, Freidhoff LR, Blumenthal MN et al: Genome-wide linkage analyses of total serum IgE using variance components analysis in asthmatic families. Genet Epidemiol 2001; 20: 340-355.

28 Blumenthal MN, Langefeld CD, Beaty TH et al: A genome-wide search for allergic response (atopy) genes in three ethnic groups: Collaborative Study on the Genetics of Asthma. Hum Genet 2004; 114: 157-164.

29 Blumenthal MN, Ober C, Beaty TH et al: Genome scan for loci linked to mite sensitivity: the Collaborative Study on the Genetics of Asthma (CSGA). Genes Immun 2004; 5: 226-231.

30 Yokouchi $Y$, Nukaga $Y$, Shibasaki $M$ et al: Significant evidence for linkage of mite-sensitive childhood asthma to chromosome 5q31-q33 near the interleukin 12 B locus by a genome-wide search in Japanese families. Genomics 2000; 66: $152-160$

$31 \mathrm{Xu}$ X, Fang Z, Wang B et al: A genomewide search for quantitative-trait loci underlying asthma. Am J Hum Genet 2001; 69: 1271-1277.

32 Teerlink CC, Camp NJ, Bansal A et al: Significant evidence for linkage to chromosome $5 q 13$ in a genome-wide scan for asthma in an extended pedigree resource. Eur J Hum Genet 2009; 17: 636-643.

33 Celedon JC, Soto-Quiros ME, Avila L et al: Significant linkage to airway responsiveness on chromosome 12q24 in families of children with asthma in Costa Rica. Hum Genet 2007; 120: 691-699.

34 Raby BA, Soto-Quiros ME, Avila L et al: Sex-specific linkage to total serum immunoglobulin $\mathrm{E}$ in families of children with asthma in Costa Rica. Hum Mol Genet 2007; 16: 243-253.

35 Levinson DF, Levinson MD, Segurado R, Lewis CM: Genome scan meta-analysis of schizophrenia and bipolar disorder, part I: methods and power analysis. Am J Hum Genet 2003; 73: 17-33.

36 Pardi F, Levinson DF, Lewis CM: GSMA: software implementation of the genome search meta-analysis method. Bioinformatics 2005; 21: 4430-4431.

37 Hermanowski J, Bouzigon E, Forabosco P, Ng MY, Fisher SA, Lewis CM: Meta-analysis of genome-wide linkage studies for multiple sclerosis, using an extended GSMA method. Eur J Hum Genet 2007; 15: 703-710.

38 Zintzaras E, loannidis JP: HEGESMA: genome search meta-analysis and heterogeneity testing. Bioinformatics 2005; 21: 3672-3673.
39 Altmuller J, Seidel C, Lee YA et al: Phenotypic and genetic heterogeneity in a genomewide linkage study of asthma families. BMC Pulm Med 2005; 5: 1.

40 Bouzigon E, Siroux V, Dizier MH et al: Scores of asthma and asthma severity reveal new regions of linkage in EGEA study families. Eur Respir J 2007; 30: 253-259.

41 Postma DS, Meyers DA, Jongepier H, Howard TD, Koppelman GH, Bleecker ER: Genomewide screen for pulmonary function in 200 families ascertained for asthma. Am J Respir Crit Care Med 2005; 172: 446-452.

42 Wilk JB, DeStefano AL, Arnett DK et al: A genome-wide scan of pulmonary function measures in the National Heart, Lung, and Blood Institute Family Heart Study. Am J Respir Crit Care Med 2003; 167: 1528-1533.

43 Soderhall C, Marenholz I, Kerscher T et al: Variants in a novel epidermal collagen gene (COL29A1) are associated with atopic dermatitis. PLoS Biol 2007; 5: e242.

44 Moffatt MF, Kabesch M, Liang L et al: Genetic variants regulating ORMDL3 expression contribute to the risk of childhood asthma. Nature 2007; 448: 470-473.

45 Bouzigon $\mathrm{E}$, Corda $\mathrm{E}$, Aschard $\mathrm{H}$ et al: Effect of $17 \mathrm{q} 21$ variants and smoking exposure in early-onset asthma. N Engl J Med 2008; 359: 1985-1994.

46 A genome-wide search for asthma susceptibility loci in ethnically diverse populations: The Collaborative Study on the Genetics of Asthma (CSGA). Nat Genet 1997; 15: 389-392.

47 Noguchi E, Yokouchi Y, Zhang J et al: Positional identification of an asthma susceptibility gene on human chromosome 5q33. Am J Respir Crit Care Med 2005; 172 : 183-188.

48 Gudbjartsson DF, Bjornsdottir US, Halapi E et al: Sequence variants affecting eosinophil numbers associate with asthma and myocardial infarction. Nat Genet 2009; 41: 342-347.

49 Wjst M: Specific IgE-one gene fits all? German Asthma Genetics Group. Clin Exp Allergy 1999; 29: 5-10.

50 Nicolae D, Cox NJ, Lester LA et al: Fine mapping and positional candidate studies identify HLA-G as an asthma susceptibility gene on chromosome $6 \mathrm{p} 21$. Am J Hum Genet 2005; 76: 349-357.

51 Maher B: Personal genomes: the case of the missing heritability. Nature 2008; 456 : $18-21$.

52 Hardy J, Singleton A: Genome-wide association studies and human disease. N Engl J Med 2009; 360: 1759-1768.

Supplementary Information accompanies the paper on European Journal of Human Genetics website (http://www.nature.com/ejhg) 